Voix et Images

voixetimages

\title{
Madeleine Gagnon : Du politique à l'intime
}

\section{Gabrielle Frémont}

Volume 8, numéro 1, automne 1982

\section{Madeleine Gagnon}

URI : https://id.erudit.org/iderudit/200362ar

DOI : https://doi.org/10.7202/200362ar

Aller au sommaire du numéro

\section{Éditeur(s)}

Les Presses de l'Université du Québec

\section{ISSN}

0318-9201 (imprimé)

1705-933X (numérique)

Découvrir la revue

\section{Citer cet article}

Frémont, G. (1982). Madeleine Gagnon : Du politique à l'intime. Voix et Images, 8(1), 23-34. https://doi.org/10.7202/200362ar d'utilisation que vous pouvez consulter en ligne.

https://apropos.erudit.org/fr/usagers/politique-dutilisation/ 


\title{
Madeleine Gagnon: Du politique à l'intime
}

\author{
par Gabrielle Frémont, Université Laval
}

Éparse, un peu partout autour de moi, sur le pupitre, le fauteuil, la table, la bibliothèque, l'œuvre presque complète de Madeleine Gagnon (mais comment réunir le tout d'un écrivain?): livres, articles de revues, commentaires, critiques, interviews, interventions de toutes sortes dans les journaux ou ailleurs, poèmes, illustrations, bref, tout ce qui, de près ou de loin, fait texte -prétexte à ma propre recherche. À tout cela, à tous ces écrits, majeurs ou mineurs selon le cas, mais tous aussi signifiants quels qu'ils soient, il s'agit de donner cohérence et forme, unité et sens ultime; tâche impossible, en réalité (car comment réunifier le sujet d'une écriture?).

Épars, un peu partout en moi, intellectuellement et affectivement, les textes lus et relus; dispersés, disséminés, éparpillés ici et là, au gré de la logique et des sentiments, perdus entre théorie, cœeur et divan... Tant il est vrai que l'écriture de Madeleine Gagnon, à la fois évanescente et durable, douce et dure, limpide et trouble, parvient à nous hanter et à nous habiter bien au-delà d'une première et même d'une deuxième lecture: tenace comme un parfum de nuit, dense comme la vie, dépouillée comme la mort. $Y$ aurait-il, dans une telle œuvre, surtout pour une femme, une fascination particulière, ou plutôt une tentation, un risque même de projection, d'identification, d'interprétation? Ou d'un peu tout cela à la fois? Comment savoir?

Depuis 1969, date de la parution de son premier livre, Les Morts-vivants, Madeleine Gagnon n'a cessé de publier régulièrement, au Québec et en France, des essais et écrits divers qu'elle intitule tantôt roman, tantôt poème, tantôt fiction, sans que l'on sache au juste la part de l'un ou de l'autre, fidèle en cela à toute une génération de nouveaux écrivains d'ici et d'ailleurs - d'écrivaines féministes en particulier - pour qui paraît s'imposer de plus en plus un refus systématique de toute forme établie, c'est-à-dire d'un genre nommé, codifié, sorte de carcan désormais rejeté au profit d'une liberté formelle plus grande. Cette volonté d'éclatement des genres littéraires accompagne, semble-t-il, de façon concomitante, et pour moi significative, une tentative d'éclatement des genres tout court, masculin et féminin. Pendant que les femmes dénoncent le phallocentrisme du discours occidental, les hommes, eux (du moins certains d'entre eux; je pense ici à Philippe Sollers ou à Philippe Haeck, par exemple), redécouvrent les vertus d'une parole dite "féminine" et caractérisée précisément par le flou, le liquide, le non structuré. On ne parle déjà plus de la spéci- 
ficité des écritures de femmes, difficile à repérer ailleurs que dans les thèmes et l'idéologie ambiante, affirme-t-on, et on en revient à cette possibilité d'écriture bisexuelle, envoûtement inégalable d'un lieu androgyne où le je peut enfin se jouer homme et femme.

Dans ce méli-mélo, pour ne pas dire mélodrame, dont se passionnent les littéraires, des voix ténues continuent à se faire entendre, contre vents, modes et marées, cherchant ailleurs que dans un présent éphémère le langage qui convient et qu'on saura enfin entendre. Madeleine Gagnon est de celles-là qui, avec une patience et un entêtement exemplaires, texte après texte, page après page, sans se lasser, sans nous lasser surtout, tente désespérément de se dire:

[...] me lever à ce devoir du jour, écrire, répondre à la demande, écrire, pour dire seulement mon refus, cette impossibilité. Cette tendance récente à me dégager de tout. De tout. De tout cela qui n'est pas essentiel. Une lenteur et un silence conjugués à l'urgence de nommer certains détails, ce qui fuit.

Au cœur de la lettre 1

Poésie. Rythme. Profondeur. Le langage de Madeleine Gagnon, dans son exigence, sa pureté (au sens chimique du terme), son hermétisme même, appelle de notre part non pas cette approche banale, superficielle et distraite qu'on prête si souvent, faute de mieux, aux textes qui nous tombent sous les yeux, mais, au contraire, cette attention flottante chère à Freud, faite de réceptivité, de réflexibilité et de respect d'une parole à la fois autre et sienne. Lecture transférentielle, en quelque sorte, que suggère elle-même l'auteure à deux ou trois reprises, et qui aurait l'avantage de transposer le texte sur la scène même du désir.

De cette "troisième oreille où s'en [iront] se perdre désormais les effets du babil stérile de la doxa ${ }^{2}$, je-narrataire, a l'écoute de tu-narratrice, dans un rapport de sympathie et de connivence, tente de retracer, au-delà des structures politiques et langagières, des positions historiques et féministes clairement établies, la trajectoire de l'écrivaine depuis une douzaine d'années, et, si possible, l'articulation d'un moi latent qui, peu à peu, émerge de l'ensemble de l'œuvre.

\section{Écran 1 : Politique et féminisme}

Du privé au public au privé, ainsi apparaît rétrospectivement, après lecture, l'ceuvre de Madeleine Gagnon.

Les premiers écrits en effet - recueil de nouvelles, récits et poèmes parus dans les Écrits du Canada français et autres ${ }^{3}$ - même s'ils abordent à l'occasion des thèmes comme ceux de la destinée de la femme ou du pays, se cantonnent plutôt, soit dans le domaine de la fiction, soit dans celui de la poésie, les deux rejoignant nécessairement, au bout du compte, l'imaginaire et l'intime. Dans Portraits du voyage, écrit en collaboration avec Jean-Marc 
Piotte et Patrick Straram le Bison ravi, qui date de 1975, de la même façon, il sera davantage question de faits personnels et de sentiments que de préoccupations collectives et sociales. A leur insu sans doute, les trois auteurs $y$ retracent l'éternelle structure œdipienne, le Bison ravi, à la pointe même du triangle, se révélant effectivement ravi d'être témoin, voyeur (sinon participant à l'occasion des ébats et débats amoureux de ses deux chers amis, Pio le fou et gentille Lionne. Si, littérairement parlant, la trame romanesque est mince ainsi que les résultats obtenus, il reste cependant que le texte nous apporte de précieux renseignements sur les recherches déjà en cours et la problématique de l'œuvre à venir de la gentille-lionne-alias-Madeleine Gagnon: problèmes de la lutte des classes, du sort des femmes, de l'importance du langage ; influence sensible de Lacan, Althusser, Cixous. (C'est dans un texte de cette dernière incidemment qu'est emprunté le pseudonyme de lionne: “Celui qui mange l'autre est lion. Où le sujet est le lion advient sa lionne ${ }^{4}$.)

La même année, Madeleine Gagnon publiera aux Herbes rouges son poème-affiche Poélitique, dans laquel elle fera part de ses convictions profondes au sujet de problèmes collectifs et culturels la préoccupant: guerre, travail, chômage, art, écriture, etc. Diefenbaker, les bombes, les prisons, les grèves, et $j$ 'en passe, tout $y$ défilera dans une forme insolite et inédite (citations, collages, montages de toutes sortes, écriture verticale, dans tous les sens) et, comme l'indique le titre lui-même, à travers un texte à la fois poétique et politique. C'est l'engagement total de l'écrivaine, dans une surenchère politicosociale d'une rare violence chez elle - avec citations de Maïakovski et de Mao comme caution de bonne foi révolutionnaire:

d'osties en tabarnacles de Montréal en Gaspésie ça finira par aboutir un jour et ne plus differer mais c'est grave dans le très grave parler encore juste écritures d'amiante de plomb pour les petitsbourgeois chauvinisme de caste haine structurelle politique intellectuels castrateurs à la solde des boss déchirement temporaire élevage qui s'amorce dans la mort quotidienne des fous prisonniers au délire refusé en ces lieux qui pourraient dépuceler la caste quand viendra la révolte de tous nos ordres symboliques enculer le pouvoir violer la patronesse masturber les ministres jusqu'à ce que mort vienne.

\section{Poélitique ${ }^{5}$}

Avec la Venue à l'écriture, publié en France en 1977, dont elle partage la signature avec Hélène Cixous et Annie Leclerc, Madeleine Gagnon emploie cette fois un langage strictement féministe, sans renier pour autant ses prises de positions politiques antérieures. C'est la Madeleine Gagnon que l'on connaît depuis: ardente féministe, défenseur acharné des droits de la femme et d'une condition féminine améliorée, celle qui ose, sans peur et sans bravade, affronter l'opinion publique, dénoncer le statu quo dans lequel les femmes sont enfermées depuis si longtemps, et réclamer pour elles un ordre et un langage 
nouveaux où elles pourraient enfin ne plus être étrangères à elles-mêmes: "Je me suis étrangère en ma propre langue [...]" 6 . Texte charnière dans l'œuvre de l'auteure, qui tente d'expliquer le pourquoi de sa lutte féministe tout en prenant conscience du même coup. dirait-on, du pouvoir des mots et de la possibilité d'un discours autre, féminin, de cette Parole de femme que vient de revendiquer Annie Leclerc tout juste au même moment, et dont elle s'inspire alors abondamment.

Depuis, que ce soit dans Retailles, Lueur ou ailleurs, Madeleine Gagnon reprendra sans cesse le même langage, clair, dru, sans compromission aucune, dans l'espoir de sensibiliser tout aussi bien les hommes que les femmes à ce discours censuré, dorénavant accessible: «Ne me parle plus d'incommunicabilité ${ }^{7}$. Le projet est infini puisqu'il ne se limite pas à la parole actuelle de la femme mais, dans un mouvement sans précédent, entend retracer toute I'histoire des femmes d'hier et d'aujourd'hui, histoire qu'on a depuis toujours occultée:

[...] des écritures qui font descendre la conscience politique de l'oppression et de l'aliénation jusqu'aux racines de son assujettissement, jusqu'aux histoires individuelles nourries des fantasmes et des désirs de l'intime, jusqu'à son inconscience même. II semble que tous les textes féministes d'ici aient fait ce même pari.

Femmes du Québec; un mouvement et des écritures ${ }^{8}$

Car il est évident que le message féministe de Madeleine Gagnon n'est pas le seul entendu et qu'il s'inscrit dans un mouvement littéraire beaucoup plus vaste qui, particulièrement au Québec, va prendre une expansion et un essor inespérés ces années-là. Alors que la solidarité des écrivaines féministes est en train de donner des fruits - "Les ventres de nos solitudes s'écrivent maintenant»-, Madeleine Gagnon continue cependant, de façon parallèle, ce que j'appellerais son voyage intérieur. Antre et Lueur, chacun à sa manière. rendent compte de ce questionnement incessant sur la vie, l'amour, la mort, de cette réflexion sans fin autour d'un je qui, qui dans le plus grand secret, tente éperdument de se dire. Puis vient le dernier très beau livre de Gagnon, Au cceur de la lettre. Tout langage politique, féministe, est ici brouillé, estompé: oui, nous voilà bien au cœur de la lettre, au cœur du cœur... Au cœur de l'intime.

\section{Écran II: Poétique et langage}

Si l'instance idéologique tient une place prépondérante chez Madeleine Gagnon - comme chez tout écrivain féministe d'ailleurs -, il serait toutefois vain de croire que là réside la spécificité de son œuvre. Pour ma part, je crois plutôt que la façon bien particulière qu'elle a de traiter le langage fait, dans une grande mesure, sa singularité et son originalité même; langage à la fois véhicule d'idées, instrument au service de la cause des femmes, bref inestimable outil de travail, mais aussi matériau vivant, irremplaçable, qui, dans sa plasticité même, permet toutes les combinaisons possibles et les jeux les plus invraisemblables. Car au-delà de tout message politique ou autre, au-delà 
même de cette volonté irréductible de prise de parole féministe-féminine, il y a cette nécessité, cette exigence intérieure, cet excès même dans l'expression, de la chose à dire, de la chose enfin dite:

Dire le flou et le dense, l'émiettement et la structure, la folie et le système. Dire, dire, ce qui s'est passé là. Risquer l'erreur et l'abandon. Ne plus nous réfugier silencieuses en ce lieu, comme des momies que l'on eût fixées avant l'heure.

Des mots plein la bouche ${ }^{9}$

Bien sûr, on ne peut rester sourd à la part de revendication qu'un tel langage recouvre, mais, de la même façon, on ne peut ignorer non plus cette dimension autre, presque de l'ordre du sacré, que laisse entendre l'intense besoin de parole à jamais inassouvi. Effet de magie, trésor, prisme, antidote à l'absence et au manque, l'écriture devient le lieu même de l'assomption du sujet, de son insertion sociale, espace privilégié entre tous, jouissance infinie sans quoi rien ne vaut plus: "Sans mots, le vide. On n'a plus le choix d'y plonger» 10 .

L'écriture - sorte de projet mythique - envahit désormais le champ même de la fiction: tantôt métaphore du corps, tantôt (déplacement suprême) corps lui-même. Corps-texte-sexe ne font plus qu'un. Ne font plus qu'Une. L'utopie est à son comble, l'écriture-corps, l'écriture-séduction fonctionnent à plein :

M'écrire et laisser couler toute mon histoire à l'infinitif. M'écrire à l'infini, me laisser fibrer dans les consonnes, me détendre dans mon corps au travers, en dessous, en dessus, tous les tissus troués. Les reprises, reprendre tous les fils à la fois. Mon corps est écritures dispersées. Les étendre, les détendre et me les ramasser. Chacune des parties, texte à constituer. Je prends pour exemple mes seins. Avec les deux mains je les travaille. Ils parlent dans mes mains qui les écrivent. Ils jouissent et me glissent entre des histoires dissemblables.

Mon corps dans l'écriture 11

Plus tard seulement viendra la totale désillusion et cette appréhension du leurre soudain, après l'euphorie d'une parole/corps féminin retrouvée: "Le corps en-deçà des mots, toujours"12. Non, l'écriture ne sera jamais qu'approximation, métonymie, tenant-lieu de; fraude essentielle certes, mais fraude quand même:

Partager ce grave espoir sans aucune illusion. Je veux que l'écriture s'approche de sa fraude et s'aime malgré tout. Je ne veux pas aimer comme on porte un drapeau. Un souvenir précis de lait de sang m'a retenue dans le langage, malgré qu'il se livrait dans le non-représentable le plus total.

Antre ${ }^{13}$

Deuil à refaire texte après texte d'un corps qui se refuse à l'écriture. Perte à combler de mots en mots, dans le mirage d'un discours structuré, organisé, de plus en plus intelligent, fort et beau. Les mots sont ciselés comme 
de purs joyaux, agencés en de savants assemblages, Travaillés, explorés, exploités jusqu'à leur extrême limite. Les jeux d'écriture les plus divers se font jour, ainsi que prend corps une constante dialectique entre fiction et théorie: «[...] la théorie, ça peut être ludique, tout autant que la fiction. En tout cas, pour moi, ça l'est. Je veux apprendre à ne plus terroriser la théorie, comme j'ai appris à ne plus terroriser la poésie ${ }^{14}$.

L'écriture s'intellectualise - ou se volatilise, selon le cas -, les mots se resserrent, le style s'en ressent. A mesure que l'énoncé se complique, s'amenuise la clarté du texte. Apparaît alors une certaine ambiguïté textuelle - ce que l'auteure appelle elle-même "l'antre-deux" - qui, par l'inconfort et l'incertitude qu'elle crée chez le lecteur, risque par moments de le perdre en cours de route.

Est-ce pour appuyer ses propres dires et ainsi se rassurer? Pour amplifier certains propos ou les annuler? Ou tout simplement rompre un discours qui menace de tourner en rond? Toujours est-il que Madeleine Gagnon, dans chacun de ses écrits ou presque, a pris l'habitude des citations - intercalées, en exergue, entre parenthèses, isolées, intégrées, de toutes les façons - et des références multiples - aux poètes, chanteurs, compositeurs, psychanalystes, féministes, amis:

[...] c'est l'infini des codes linguistiques psychiques l'infini des dès hazart mallarmé mozart cixous stockhausen haeck straram grammes pour grammaire apprentissage tisser les trames réglées du début à la fin contraintes transgressions ou pour mouvance désir tendresse et toute la mode et la modernité ensemble [...]

Antre ${ }^{15}$

La pratique de l'intertexte (pratique devenue courante chez maints écrivains d'aujourd'hui), en plus de permettre une grande ouverture d'écriture et d'esprit, procure au lecteur le précieux avantage de redécouvrir - ou de découvrir, soyons modeste - textes et auteurs connus et méconnus, Freud, Lacan (depuis peu sujet barré...) et Derrida y côtoyant allègrement aussi bien Marguerite Duras que Claire Lejeune ou Félix Leclerc:

Et tant que ça me plaira, il y aura des parenthèses.

Et tant que ça me plaira, des citations.

Et tant de textes et de paroles.

\section{Retailles; complaintes politiques ${ }^{16}$}

Les propres textes de Madeleine Gagnon n'échappent d'ailleurs pas à la règle et, de façon récurrente et répétitive, refont surface eux aussi d'un écrit à l'autre. Comme s'il fallait sans cesse répéter les choses... Ou ne rien perdre... Des autres, de soi, du rêve, de la réalité, du fantasme, du désir. Et des femmes. 


\section{Lueur I: Femme et psychanalyse}

De tous les sujets abordés par Madeleine Gagnon, incontestablement, c'est la femme qui occupe le champ le plus vaste; et l'intérêt de ses textes est sûrement doublé par l'intérêt même porté à la condition féminine - réflexion, volonté d'approfondissement, ou sympathie tout simplement de la part du lecteur. Dans une approche à la fois historique, onirique, je dirais presque fantasmatique, la mémoire se met en marche, omniprésente, irréfutable et implacable, afin de découvrir ce qui, de la femme, est resté enfoui sous les décombres d'un discours phallocrate en train d'agoniser. Les images qui défilent sous nos yeux, comme dans un film noir, (images de misère et de drame la plupart du temp), sont souvent culpabilisantes et accusatrices, de l'ordre du ressentiment en tout cas. En fait, il s'agit surtout de sensibiliser l'autre - tantôt l'homme, tantôt la femme, tantôt les deux — au sort tragique fait aux femmes:

Et les classes: Qu'est-ce que t'en fais, des classes? En? es-tu dedans, oui ou non? Telle quelle, ton écriture ne peut pas s'articuler. À rien, tu comprends, à rien. Et les femmes, qu'est-ce que t'en fais, des femmes? En? En?

\section{Lueur: roman archéologique ${ }^{17}$}

Mais, à vrai dire, le texte prend surtout l'allure d'une course à rebours, presque chronomètre en mains, vers l'origine de nos destinées de femmes, vers ces temps immémoriaux où, sans doute, sur quelque plaquette ancienne, ont été inscrites les causes premières de nos frustrations et de nos servitudes, les traces ultimes de notre profonde aliénation. A cet égard, le sous-titre de l'important livre Lueur (important tant dans son contenu que dans sa forme) est éloquent, l'expression roman archéologique ne laissant planer aucun doute quant au sens de la recherche entreprise. II faut, une fois pour toutes, et grâce à la magie de l'écriture, sortir de l'ombre cette femme inconnue, effacée, volée et violée par l'Histoire, que seules nos mémoires à l'affût peuvent nous restituer, «Faire un lieu-dit de son absence». Le langage, de type psychanalytique, chercher à créer, à recréer sans cesse l'objet perdu - et bien plus le sujet perdu:

Dis-leur qu'il est possible de remonter, de revenir, de remonter encore sans se perdre, ou plutôt, dis-leur que pour se perdre parfois il faut revenir très loin, plus loin que ses propres traces, là où l'on avait pas encore osé inscrire $[\ldots]$.

Lueur ${ }^{18}$

Morte ou vivante, mère ou grand-mère, jeune, vieille ou à venir, la figure de mère prendra définitivement la première place. Mère dont la fille n'a jamais su trop comment se déprendre, se dépêtrer; mère qu'on lui a maintes fois proposée comme modèle (d'abnégation, de résignation, de soumission, cela va sans dire); mère, qu'avec Madeleine Gagnon, on apprend à regretter, à re-aimer, à écouter: «Parle parle grand-mère, on a besoin de ton savoir pour continuer la vie»19. Mère, reine du foyer, mais bien plutôt reine de tous les 
malheurs inimaginables, statue de sel et de larmes. Les plus beaux textes de Madeleine Gagnon seront inspirés par le. souvenir de cette tendre image maternelle:

Laissez-moi pleurer, disait-elle. Une mère. Laissez-moi pleurer toutes les larmes de mon corps et des corps que je berce. Laissez-moi pleurer ça ne vous fait pas mal et je ne crierai plus [...]. Laissez-moi me lamenter, me rouler dans ma peine et regretter d'avoir enfanté tous ces enfants, au large cœur pourtant, qui s'entredéchirent sous mes yeux. Laissez-moi contempler mon malheur, $m$ 'en induire, $m^{\prime} y$ vautrer, laissez-moi me saouler de mes larmes. Laissez-moi $\mathrm{m}^{\prime} \mathrm{y}$ laisser couler vive, si ça me chante. Folle, folle mère, partie ou ne sait où au bout de ses eaux. Puis laissez-moi mourir de vous aimer quand même.

Lueur ${ }^{20}$

Après la mère, la scur. Madeleine Gagnon reste l'une des seules écrivaines d'ici, à ma connaissance, à avoir osé aborder le thème de la sororité. Comme si les autres, de peur de passer pour rivales, mesquines, ou je ne sais quoi, craignaient d'affronter un tel sujet, se contentant de chanter, seules ou en chœur, les merveilles de la solidarité et de l'amitié féminines. Tout au contraire, Retailles, écrit en collaboration avec Denise Boucher, nous fait part, avec franchise mais non sans quelque réticence, d'une expérience vécue, à la fois pénible et enrichissante (du moins sur le plan littéraire puisque le livre a été un succès) d'éclatement d'un groupe de femmes. Rancœur, animosité, difficultés personnelles, le texte, pour une grande part, est une triste histoire de pots cassés, apparemment difficiles à recoller. Mais malgré la brusquerie et la brutalité même de certains écrits («Je dirai ce que je peux quand je peux à qui je peux à qui je veux plus de ces liaisons piégantes collectives des jeux de vérité...» $)^{21}$, on sent le désir profond de pactiser avec «elles», et surtout le regret de n'avoir pu éviter l'affrontement:

Je ne crois absolument plus aux groupes dits de conscience: ils constituent sans doute le piège le plus raffiné du féminisme actuel. Comment, issue de la psychanalyse, ai-je pu l'ignorer à ce point? Pourquoi? Que voulais-je éviter là?

Retailles 22

Reste l'inévitable solitude, seule créatrice, à bien y penser. C'est, une fois de plus, la remise en question des rôles féminins et masculins, des oppositions femmes-hommes, de l'identité peu assurée de l'une par rapport à l'autre; utérus-femme, phallus-homme, discours hystérique, discours obsessionnel, selon que l'on est une ou un, menstrues, accouchement, sang, lait, sperme, délire, folie, raison, logique: rien n'échappe à l'œil inquisiteur et à la réflexion lucide de l'auteure.

Quant à la psychanalyse, dont il est si souvent fait mention, on la dénie ou on la magnifie ou les deux à la fois, selon le cas:

Vous êtes là, silencieuses, à l'attention flottante de vos propres dérives. Comme moi vous descendez ce long fleuve qu'un jour vous aviez 
remonté, pour en saisir la source et le sens. Nos itinéraires s'entrecroisent en ces voyages réciproques et parallèles. [...] Quel est l'enjeu de ce désir: Avons-nous, quelque part, au cours de nos analyses respectives, laissé un immense blanc que la psychanalyse elle-même ferait paraître pour indiquer seulement son impuissance à combler?

Lueur lou Pré-texte) $)^{23}$

Mais, de toute façon, ne sommes-nous pas, depuis le début, en plein savoir analytique? D'une part, le glissement du textuel au sexuel, que ce soit à travers les figures maternelles, la confrontation des images corporelles hommes-femmes et des discours respectifs de chacun, s'est opérée comme allant de soi; alors que, d'autre part, le langage lui-même, dans une mystérieuse alchimie - celle même de l'art véritable - s'est comme fondu et confondu entre fantasme et réalité, imaginaire et mots. Atemporel, aspatial, aux frontières toujours de la conscience et de l'inconscience, n'est-ce pas, en un sens, le discours même de l'analysant?

On pourrait, de façon plus générale, parler de discours du rêve tout simplement. Les phénomènes de dépersonnalisation et de déplacement qui apparaissent si souvent dans le texte, ainsi que la continuelle tentative de projection et d'identification entre personnages et instances énonciatrices, créent d'ailleurs cette espèce d'illusion narrative et cette dramatisation propres au langage du rêve. Chez Madeleine Gagnon, vie et mort se frôlent, passé et présent s'affrontent, colère, violence et vérité surgissent, comme dans un cauchemar. Amour, douceur, tendresse et illusion refont surface, comme dans un rêve. "Je songe que le rêve s'écrit".

\section{Lueur II: Je}

Comme des ombres chinoises se projetant sur les écrans plus ou moins opaques des discours idéologiques et théoriques, sont donc apparues les sombres silhouettes des femmes souffrantes d'hier et d'aujourd'hui : femmes violées, excisées, bafouées dans leurs droits et dans leur cœur. Mais à travers ces tableaux en noir et blanc - corps et chagrins surexposés - à travers cette représentation multiple de visages féminins, se profile aussi dans l'espace textuel, un moi fragile, craintif, fuyant même, à tout instant en péril de disparaître, un je qui, dans la plus grande discrétion, souvent à demi-mots, tente tout à la fois de se cacher et de se révéler. Les «elles» en effet glissent sans cesse aux «elle» aux «nous» aux «tu» aux «je»; tous les pronoms personnels y passent, chacun d'eux participant à sa façon au même sujet narré :

Qui donc, hier, luisait encore dans tes mots? Les voix d'elles, nombreuses, incertaines? Tu te souviens, cet espoir, ces nombrantes de moi ressurgies, on ne savait trop comment.

Lueur ${ }^{24}$

Les contours des êtres et des choses se fondent alors dans une espèce de tout imprécis et flou, à la limite du représentable. Nous sommes ici en plein impressionnisme. Et, paradoxalement, nulle part ne sera aussi sensible la 
présence possible d'un je, impalpable, indécidable, mais qui, à force de se dire et de ne pas se dire, finit par s'inscrire et se faire entendre.

Dans cette dépersonnalisation de soi et des autres, dans la fragmentation et la déstructuration de plus en plus visible du discours, tant au niveau de l'énonciation qu'au niveau de la fable elle-même, se fait sentir l'influence certaine de Marguerite Duras et, à un degré moindre, celle de Clarice Lispector. Un peu comme ces deux femmes-écrivains, au sommet d'une écriture qui aura marqué la littérature des femmes ces dernières années, Madeleine Gagnon semble être arrivée, du moins dans la deuxième partie de son œuvre, c'est-àdire avec la parution d'Antre, à une expression du désir, à une recherche d'intériorité et d'approfondissement de soi que peu d'écrivaines d'ici ont atteintes (sauf peut-être France Théoret qui dans son inlassable exploration de la mémoire et de l'inconscient, me paraît aller aussi loin). Les passages les plus marquants de Gagnon sont empreints de ce langage d'irréalité à la Duras, et de ce ton indéfinissable qui, sans aucun doute, font les grands textes. Mais alors que Lol-la-folle, du Ravissement de Lol V. Stein, se perd dans le plus complet délire, Elle de Lueur tente de se retrouver dans l'intelligence des mots; langage de la folie ou des hiéroglyphes, champ de seigle ou de blé, sens ou non-sens, l'effet reste étrangement le même:

Un pré de blé. En plein milieu de la forêt touffue, un pré de blé. Immense. Je ne l'aurais pas cru possible. Elle est allongée de tout son long dedans, une femme, comme endormie, la tête sur une ardoise plate où c'est écrit, hiéroglyphes. Des lettres sont gravées dont elle ne comprend ni la disposition ni le sens. Elle se repose et ne semble pas vouloir chercher ; de son index, elle balaie la poussière de blé sur hiéroglyphes. Elle souffle sur l'ardoise. Émergent des mots signifiants jamais appris nulle part. Et comment, si ces mots ne furent pas appris, peut-elle prétendre au sens?

Lueur $^{25}$

Peut-on dans de tels textes, parler d'autobiographie? Si, d'une part, le genre autobiographie suppose une identité assumée au niveau de l'énonciation, et si, d'autre part, le lecteur a toutes les raisons de soupçonner qu'il y a coïncidence entre auteur-narrateur-personnages, on ne voit pas comment la réponse pourrait être négative. En ce qui regarde Madeleine Gagnon en tout cas, il est clair que, dans une large mesure, le je énoncé, ainsi que nombre d'autres pronoms (elles, elle, nous, tu, etc.), sont tout à fait siens; les noms de ses enfants, Charles et Christophe (ou leurs initiales $C h$. Ch.), les noms d'amis, de lieux ou autres, en sont d'ailleurs des indicateurs de plus.

Mais le projet autobiographie est loin d'être toujours aussi clair puisque. à tout moment, le texte bascule dans la fiction pure. Mixité des genres qui, chez Gagnon, va jusqu'à la mixité des discours mêmes, l'écriture nous entraînant à tout propos vers un ailleurs indéchiffrable, à la fois dans le langage et en-deçà, en cette zone innommable et impénétrable de l'Autre. Depuis l'avènement de Freud et de la psychanalyse, il serait cependant difficile de croire qu'on peut atteindre quelque vérité intime que ce soit par le seul truchement du discours 
logique et cohérent; et c'est bien plus à travers l'imaginaire, les rêves, les délires et fantaisies de toutes sortes, qu'on peut enfin arriver à percer tant soit peu l'armature du moi: «Cette fiction est mienne, tout à fait, je ne suis même qu'elle ${ }^{26}$. Sombrer du côté du fantasme, se laisser aller à une certaine dérive, nager entre deux eaux au risque de s'y noyer, frôler même les bords de la folie et de la mort, n'est-ce pas là la tentation et la fascination suprêmes?

Le moi, tantôt se multiplie («J'étais comme dix-huit femmes ensemble ${ }^{27}$ ), enfle et s'étend aux dimensions mêmes de l'univers ( "... pourvu que je proclame autant ce que je suis d'océans, de liquides multiples» 28 ), tantôt, au contraire, se rétrécit, se fissure et semble éclater de toutes parts: tissu troué... échancrure... mots fendus... blessure démesurée... A cet égard, les métaphores récurrentes de couture - repriser, faufiler, tisser, coudre, découdre -, que le titre de Retailles met particulièrement en relief, illustrent on ne peut mieux ce morcellement du sujet et vient renforcer l'image d'un je en mille miettes qui, de maux en mots, paraît courir vers sa propre perte:

dans cette brèche ténue lucidité-déraison où folie-démence n'a pas de place, je suis devenue translucidité, une épilepsie douce en pleine lumière jouissance et n'ai eu qu'à détruire ce que j'ai aimé me détruisant.

Antre 29

Après la destruction, le rien, le vide. «Dans ce vide, soudain». Je risque de n'être plus que ce vide, que ce rien, de n'être plus personne. Seule l'écriture peut colmater la faille profonde : écriture-phare, écriture-bouée. S'entremêlent alors dans la mémoire désolée les lieux perdus de l'amour et des jours heureux. La mort rôde. Déjà.

[...] d'avoir appris l'inévitable carence, je te dis, dans la mesure violente du rien. J'écris le cri seul, il y a des cris heureux, les cris de toutes naissances, avec cette nécessité parfois d'accoucher de la mort. Ça se donne des airs de fantôme, ça fait du bruit d'ossements rouillés, on dirait. Tourne la page, cela ne te regarde plus.

Au coeur de la lettre 30

L'amour, la mort, la douleur de vivre, ne sont plus soudain le lot de l'autre. mais le sien propre. Chose étrange cependant, ou difficulté toujours de trouver sa propre identité, c'est à partir de la voix d'une autre que retentira le cri essentiel: «J'aimerai quiconque entendra que je crie que je t'aime» (Marguerite Duras, Les Mains négatives) $)^{31}$. Puis, l'écriture fera place à la gravure. Il était sans doute inévitable que dans son dernier livre, Madeleine Gagnon en arrive à l'expression picturale, cette parole de silence. Car du cri au silence, de l'amour à la mort, y a-t-il autre issue?

1. Madeleine Gagnon, Au coeur de la lettre; Poésie, Montréal, Madeleine Gagnon \& vlb éditeur, 1981, p. 81.

2. Madeleine Gagnon, «Si j"écris", in Au fond des yeux; 25 Québécoises qui écrivent, Photographies de Kéro, Montréal, Kéro \& les Éditions Nouvelle Optique, 1981. p. 30 . 
3. Voir Les Morts-vivants, recueil de nouvelles, H.M.H., Montréal, 1969, «La Laide», "Wilfrid le quéteux" (1969) et "L'Autre Bord de la rivière" (1970), in Écrits du Canada français, nos 25 et 29.

4. Madeleine Gagnon, "Amour parallèle», in Portraits du voyage, (Jean-Marc Piotte, Madeleine Gagnon, Patrick Straram le Bison ravi), Montréal, Les Éditions de l'Aurore, 1975, p. 47.

5. Madeleine Gagnon, Poélitique, Les Herbes rouges, $n^{\circ} 26$, Montréal, février 1975. non paginé.

6. Madeleine Gagnon, "Mon corps dans l'écriture», in La Venue à l'écriture, (Hélène Cixous, Madeleine Gagnon, Annie Leclerc), Paris, Union Générale d'Éditions, Coll. 10/18, 1977, p. 71.

7. Denise Boucher / Madeleine Gagnon, Retailles; Complaintes politiques, Montréal, Éditions l'Étincelle, 1977, p. 29.

8. Madeleine Gagnon et Mireille Lanctôt, «Femmes du Québec; un mouvement et des écritures», in Magazine littéraire / Spécial Québec), $n^{\circ} 134$, Paris, mars 1978, p. 99.

9. Madeleine Gagnon, «Des mots plein la bouche», in La Barre du jour (Le Corps les mots l'imaginaire), nos 56-57, Montréal, mai-août 1977, p. 142.

10. Ibid., p. 147.

11. M.G., La Venue à l'écriture, p. 84.

12. M.G., Au coeur de la lettre, p. 74.

13. Madeleine Gagnon, Antre, Les Herbes rouges, nos 65-66, Montréal, juillet-août 1978 , p. 34.

14. Madeleine Gagnon, Texte in Liberté (La femme et l'écriture), Actes de la rencontre québécoise internationale des écrivains, $n^{\circ}$ 106-107. Montréal, juillet-octobre 1976, p. 249.

15. M.G., Antre, p. 16.

16. M.G., Retailles, p. 98.

17. Madeleine Gagnon, Lueur; roman archéologique, Montréal, Madeleine Gagnon \& vlb éditeur, 1979, p. 52.

18. Ibid., pp. 95 96 .

19. Ibid., p. 80 .

20. Ibid, pp. 64-65.

21. M.G., Retailles, p. 72.

22. Ibid., p. 149.

23. Madeleine Gagnon, "Lueur" ou "Pré-texte», in Son Psychanalyste, Revue Interprétation, $n^{\circ} 21$, Montréal, printemps 1978, pp. 69-70.

24. M.G., Lueur, p. 31 (souligné par nous).

25. Ibid., p. 58.

26. Ibid., p. 79.

27. M.G., Portraits du voyage, p. 79.

28. M.G., La Venue à l'écriture, p. 72.

29. M.G., Antre, p. 33.

30. M.G., Au cour de la lettre, p. 64.

31. Ibid., p. 49. 